\title{
Symmetrical Trimalleolar Fractures of the Bilateral Ankles: A Case Report
}

\author{
Hiroyuki Nagasawa a, b, Naohisa Miyakoshia, Yuji Kasukawa, \\ Masaaki Takeshima ${ }^{a}$, Yoichi Shimada ${ }^{\mathrm{a}}$
}

\begin{abstract}
A rare case of symmetrical trimalleolar fractures of bilateral ankles has been described. A 54-year-old man fell off a motorbike on a snow covered road with inversion of bilateral ankle joints symmetrically, and sustained symmetrical trimalleolar fractures of bilateral ankles. The patient underwent osteosyntheses on bilateral ankles and the post-operative course was uneventful. The patient had no complaints at 5 years following surgery. Probable mechanism of the fracture is discussed.
\end{abstract}

Keywords: Trimalleolar fracture; Ankle; Symmetric

\section{Introduction}

Ankle fracture is a common injury occurring under variety of conditions such as falls, sports, and traffic accident irrespective of age and sex. It has been reported that $20-30 \%$ of ankle fractures occur in the elderly people each year [1]. However, bilateral trimalleolar fracture of the ankle in a symmetric pattern that has not previously been reported in the literature to our knowledge. We report here an extremely rare case of symmetrical trimalleolar fracture of the bilateral

Manuscript accepted for publication November 24, 2011

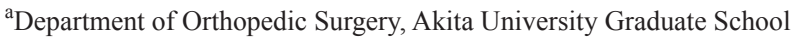
of Medicine, Akita, Japan

${ }^{\mathrm{b}}$ Corresponding author: Hiroyuki Nagasawa, Department of Orthopedic Surgery, Akita University Graduate School of Medicine,

1-1-1 Hondo, Akita, 010-8543, Japan.

Email: nagasawa@med.akita-u.ac.jp

doi:10.4021/jmc440w ankles. The mechanism of the injury was also discussed.

\section{Case Report}

A 54-year-old man with a history of integrated psychological dysfunction for about 30 years fell off a motorbike in January 2003 . When he was trying to start at an intersection on a snow-covered road, the front wheel of his motorbike lifted and the machine flipped over backwards. As a consequence, he fell on the ground hitting the bilateral feet with inversion of the ankle joints. He was unable to stand due to severe bilateral ankle pain. On admission to our hospital, the bilateral ankles showed obvious swelling and deformation. The body weight of patient was $80 \mathrm{~kg}$ and body mass index was 30.74 $\mathrm{kg} / \mathrm{m}^{2}$.

Antero-posterior and lateral radiographs demonstrated the trimalleolar fractures with slight dislocation of the bilateral ankles. These bilateral ankle fractures showed symmetrical features, the spiral oblique fracture line of the fibula that runs from the anterior distal margin up to the posterior superior cortex combined with posterior malleolar fragments and fracture of the medial malleolus respectively (Fig. 1A-D). Both fractures were diagnosed as stage 4 supination-external rotation type fracture in Lauge-Hansen's classification [2].

Surgery was performed 4 days after the injury. The fractured fibulae were fixed with a plate and screws on the right side and stabilized with screws alone on the left side. Both fractured medial malleolus were repositioned and then fixed with Kirchner wires and tension band wiring.

The patient underwent short-leg casting for 2 months without weight bearing. After removal of the cast the patient was allowed to walk with crutches and the postoperative course was uneventful. The bone union was complete by 3 months with no loss of correction in anatomical alignments (Fig. 2A-D). At follow-up 5 years later, he had no symptoms.

\section{Discussion}

Ankle fractures are a very common traumatic injury. The incidence of ankle fracture was reported to be 107 fractures per 


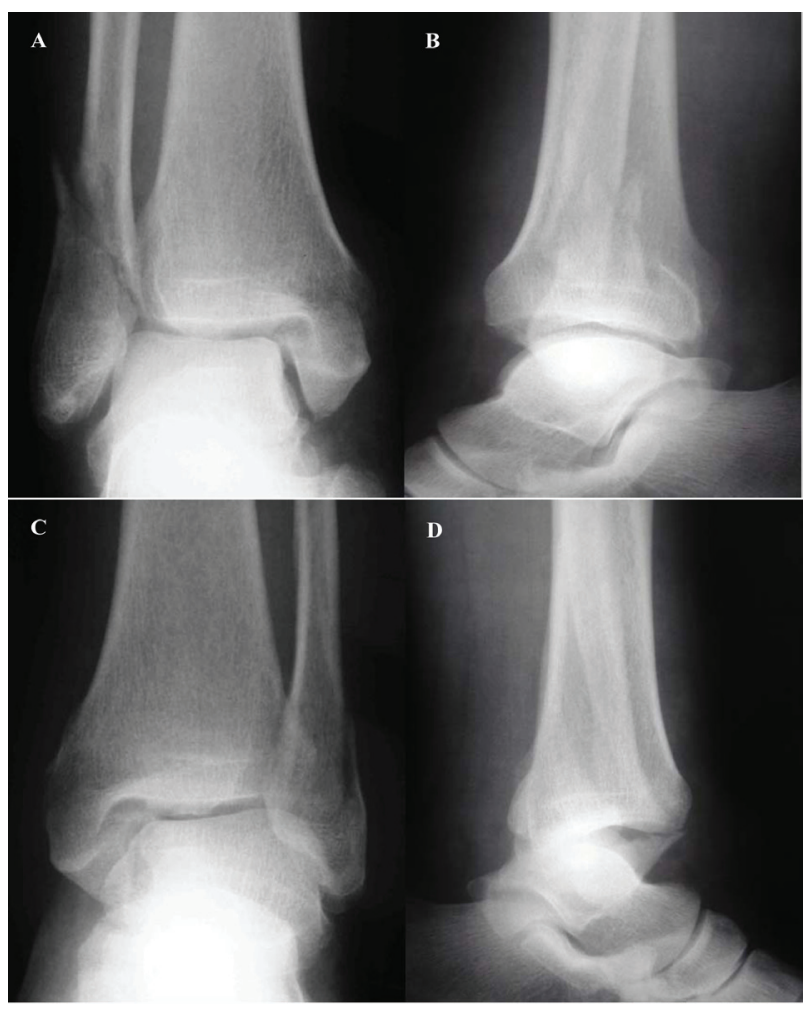

Figure 1. Preoperative anteroposterior $(A)$ and lateral $(B)$ right ankle. Preoperative anteroposterior (C) and lateral (D) left ankle.
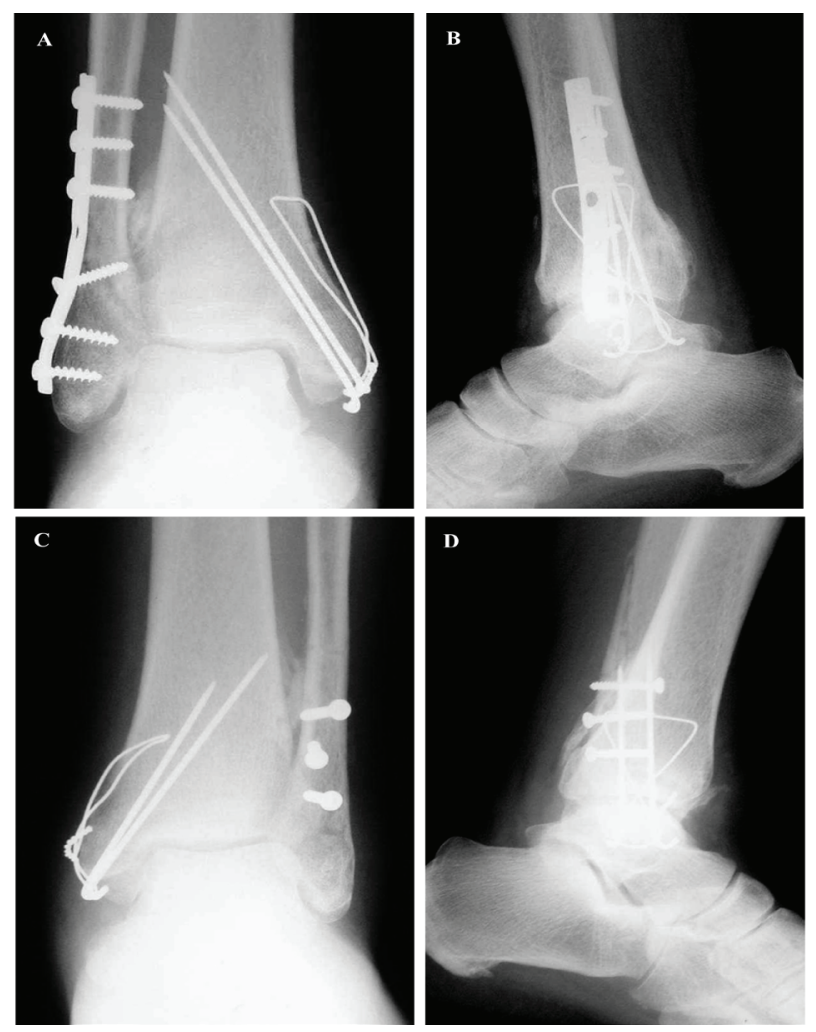

Figure 2. Anteroposterior and lateral radiographs three months after surgery showing complete union of the fractures. $A$ and $B$, right side; $C$ and $D$, left side. 
100000 person-years [3]. Koval et al reported that $14.2 \%$ were trimalleolar fractures in the investigation into 33704 ankle fractures [4]. Bilateral trimalleolar ankle fractures are rare and we found only one previous article on this topic. McDaniel and Wilson described a total of 51 trimalleolar ankle fractures in 50 consecutive patients, including only one patient with bilateral trimalleolar ankle fractures. However, they do not describe in detail whether the bilateral trimalleolar fractures were the symmetrically type or simultaneous incidence in their patient [5]. Bilateral symmetrical trimalleolar ankle fractures have not previously been reported in the literature to the best of our knowledge. In the present case, the preoperative radiographs demonstrated that fractures of the bilateral ankles are both classified as stage 4 of the supination-external rotation type in the Lauge-Hansen's classification [2]. Surgical management was successful for the present case.

The mechanism of this extremely rare occurrence should be noted. In the present case, the patient reported that the toes of both feet touched the ground in a symmetrical position when he fell from the motorbike. During traumas such as traffic accidents, sports, and falls, completely symmetrical positions of the ankles seldom occur. In this case, full body weight was loaded bilaterally and equally on both ankles in inversion at injury. Consequently, the amount of force on both feet simultaneously sprained the bilateral ankles in the direction of supination-external rotation.

\section{Conclusion}

In summary, we report an extremely rare case of symmet- rical-bilateral trimalleolar fractures of ankles. Symmetrical and simultaneous forces applied to the ankles are thought to be a cause of these fractures. Surgical management resulted in a good clinical outcome for the present case.

\section{Conflict of Interest}

None.

\section{References}

1. Salai M, Dudkiewicz I, Novikov I, Amit Y, Chechick A. The epidemic of ankle fractures in the elderly--is surgical treatment warranted? Arch Orthop Trauma Surg. 2000;120(9):511-513.

2. Lauge-Hansen N. Fractures of the ankle. IV. Clinical use of genetic roentgen diagnosis and genetic reduction. AMA Arch Surg. 1952;64(4):488-500.

3. Jensen SL, Andresen BK, Mencke S, Nielsen PT. Epidemiology of ankle fractures. A prospective populationbased study of 212 cases in Aalborg, Denmark. Acta Orthop Scand. 1998;69(1):48-50.

4. Koval KJ, Lurie J, Zhou W, Sparks MB, Cantu RV, Sporer SM, Weinstein J. Ankle fractures in the elderly: what you get depends on where you live and who you see. J Orthop Trauma. 2005;19(9):635-639.

5. McDaniel WJ, Wilson FC. Trimalleolar fractures of the ankle. An end result study. Clin Orthop Relat Res. 1977(122):37-45. 